\title{
What is learned in knowledge-related categories? Evidence from typicality and feature frequency judgments
}

\author{
THOMAS L. SPALDING \\ University of Iowa, Iowa City, Iowa \\ and \\ GREGORY L. MURPHY \\ University of Illinois, Urbana, Illinois
}

\begin{abstract}
When a category's features are tied together by integrative knowledge, subjects learn the category faster than when the features are not directly related. What do subjects learn about the category in such circumstances? Some research has suggested that the subjects can use the knowledge itself in performing the category learning task and, thus, do not learn the details of the category's features. Two experiments investigated this hypothesis by collecting feature frequency estimates after category learning. The results showed that integrative knowledge about a category did not decrease subjects' sensitivity to feature frequency-if anything, knowledge improved it. A third experiment found that integrative knowledge did reduce sensitivity to feature frequency in typicality ratings. The results suggest that knowledge does not inhibit the learning of detailed category information, though it may replace its use in some tasks.
\end{abstract}

Concepts are at the very center of cognition. They allow us to make numerous inferences about entities (objects, events, etc.) that we have never encountered before. Shared concepts allow us to communicate with each other and exchange information. One reason why concepts can be so helpful is that members of the same category tend to share many properties, whereas members of different categories tend to share relatively few properties (e.g., Rosch \& Mervis, 1975). Thus, once one knows that a particular thing is a member of a certain category, one is in a position to infer a number of its unseen properties (Smith \& Medin, 1981).

An important part of category learning, then, is learning which properties each category has (see Komatsu, 1992, for a review of research on the structure of concepts). But it has recently become evident that a person's background knowledge also plays an important role in how categories are learned and used (Medin, 1989; Murphy, 1993; Murphy \& Medin, 1985; Murphy \& Spalding, 1995; Pazzani, 1991; Spalding \& Murphy, 1996; Wisniewski, 1995). What counts as a feature of a category and which features are likely to be important are aspects of category acquisition that might be strongly influenced by

Parts of this research were supported by NIMH Grant MH41704 to the second author. The authors thank Lisa Oakes and Jodie Plumert for helpful comments on an earlier version of this paper. The authors also thank Brad McDowell and Victoria Phillips for their help in data collection. Correspondence concerning this article should be addressed to T. L. Spalding, University of Iowa, Department of Psychology, 11 Seashore Hall East, Iowa City, IA 52242-1407 (e-mail: thomas-spalding @uiowa.edu). the learner's background knowledge (see Heit, 1997, for discussion). Therefore, understanding how background knowledge affects category learning is critical to understanding concept acquisition, representation, and use. The present experiments focused on the effect of knowledge on category learning processes for stimuli that have easily identifiable features.

There have been several demonstrations that categories are more easily learned when the learner has appropriate background knowledge (see, e.g., Murphy \& Allopenna, 1994; Murphy \& Kaplan, 1997; Pazzani, 1991; Spalding \& Ford, 1998; Spalding \& Phillips, 1998a, 1998b; Wattenmaker, Dewey, T. D. Murphy, \& Medin, 1986). For example, Pazzani (1991) gave some of his subjects explanations of categories before they tried to learn them. Subjects who were given an explanation learned the categories much more quickly than subjects who were not given an explanation. Similarly, Murphy and Allopenna (1994) showed that when the features of a category could be connected through a prior knowledge structure (not described to subjects in advance), subjects were much faster at learning the category.

In short, it is now well documented that when knowledge is available to aid subjects in learning categories, learning is in fact considerably faster. What is not so clear, however, is the mechanism by which this occurs and what the product of the learning process is. That is, we do not know exactly what people are learning about the categories while they are making use of background knowledge. There are two general possibilities about how knowledge could be acting in these situations. One possibility is that subjects learn a relevant knowledge struc- 
ture (or in some cases are told this in advance, as in Pazzani's, 1991, experiment) and rely primarily on this to categorize new items. Thus, it is the knowledge structure itself that is primarily learned and used. A second general possibility is that knowledge is aiding the acquisition of the statistical structure-the learning of which features or exemplars are associated to which categories. This difference is important, because it suggests two somewhat different representations that result from knowledge-based category learning. The first suggests that the result of learning is a knowledge structure rather than details of the category's features. The second possibility suggests that the concept representation contains many specific details, even though the general knowledge has allowed faster learning.

Let us explain this distinction further with an example. Murphy and Allopenna (1994) compared learning of categories in which the features could be easily integrated through background knowledge to categories in which the features could not be easily integrated. For example, some subjects learned about two kinds of vehicles. In one condition, the properties of the items could be those appropriate for either an arctic vehicle (in one category) or a jungle vehicle (in the other category): One category contained such features as "goes in jungles" and "is made in Africa," whereas the other contained such features as "goes on glaciers" and "is made in Norway." Such integrated categories were much more easily learned than nonintegrated categories, which consisted of unrelated vehicle properties, such as "has seat belts" and "is blue." That is, the learners found it much easier to categorize the instances correctly when the categories corresponded to coherent background knowledge than when no such knowledge was available.

The question, then, is exactly what subjects learned in these two conditions: How did the knowledge influence the category representation? Although it is possible that the background knowledge made it easier to learn the category structures, it could be that learners were only figuring out the relevant knowledge and then using that. If subjects had simply inferred that "category A is a kind of jungle vehicle, and category $B$ is a kind of cold-weather vehicle," they could have performed perfectly in the categorization tests even if they had not learned anything else about the structure of the categories, simply by deciding whether each test item was a kind of jungle vehicle or a kind of cold-weather vehicle. Indeed, it is possible that the learners knew very little about the statistical structures of the two categories - that is, what the features of each category were, how frequent they were, and how predictive of the category they were. Thus, the improvement in categorizing the instances in the integrated condition does not necessarily mean that those subjects learned the statistical structure of the categories better than subjects in the nonintegrated condition. (This point is true of many experiments investigating effects of background knowledge.) In contrast, subjects who learned categories without the benefit of such knowledge presumably did learn about the detailed category structure, because there was no other way for them to acquire the categories.

Of course, these two possibilities are being stated in rather extreme forms, and it is possible that a weaker version of one of them is true. For example, it seems unlikely that people learn nothing about the features of the category when there is background knowledge available. Perhaps some learning of the detailed structure takes place before the relevant knowledge has been identified. However, it could be that the background knowledge does not improve this learning-or even interferes with itbecause it provides a way of categorizing stimuli that does not require learning the detailed category structure. In fact, there is some evidence for this position. Murphy and Allopenna (1994) found that subjects learning the integrated categories knew less about the structure of the categories than did subjects learning the nonintegrated categories, even though subjects in the integrated condition were much better at classifying the examples. In their Experiments 2 and 3, each category included both high- and low-frequency features. After learning the categories, subjects were asked to categorize a number of test instances. For subjects who learned the nonintegrated categories (features not related by knowledge), test items containing the low-frequency features were categorized more slowly than test items containing the high-frequency features. However, for subjects who learned the integrated categories, test items containing the low-frequency features were categorized nearly as quickly as those containing the high-frequency features. Furthermore, when subjects rated the typicality of instances, there were large differences between the high- and low-frequency features in the nonintegrated condition, but there were quite small differences in the integrated condition. That is, subjects in the nonintegrated condition were highly sensitive to the frequency of the features, but subjects in the integrated condition were relatively insensitive to feature frequency in categorization and typicality judgments.

Murphy and Allopenna (1994) proposed that one of the advantages of having background knowledge is that learners need not engage in the laborious process of learning the statistical regularities of the category. Instead, they can use their integrative knowledge to make decisions (such as classifying new examples) that would otherwise require learning the detailed statistical structure of the categories. Thus, one of the benefits of background knowledge is that it shortcuts the necessity for further learning.

We will refer to the idea that the availability of background knowledge decreases sensitivity to empirical category information as the empirical insensitivity hypothesis. Similar arguments can be found throughout the broader memory literature. Knowledge that relates material in a text may result in better memory for meaning (gist) but worse memory for details of the material (e.g., Bransford \& Franks, 1971; but see Craik, Moscovitch, \& McDowd, 1994, and Murphy \& Shapiro, 1994). In addition, research on scripts and schemata has clearly shown that knowledge structures can sometimes override the memory for the actual materials presented to subjects 
(e.g., Bower, Black, \& Turner, 1979; Brewer \& Treyens, 1981). Thus, it may be a more general property of memory that knowledge can short-circuit the learning of specific details.

However, the proposal that knowledge inhibits more detailed learning is problematic in some respects as well. Although knowledge of a general domain (such as animals or computers) can certainly be helpful in learning about a new kind of thing, it is rare that prior knowledge tells us everything or even most of the things we need to know about a new category. If one were to rely too much on prior knowledge, ignoring the empirical information available through experience with the category, one would be unable to learn many new things.

Is there any evidence suggesting that the empirical insensitivity hypothesis is incorrect? Recently, Wisniewski (1995) showed that category learners can make use of both background knowledge and empirical experience with members of a new category. In particular, Wisniewski showed that learners categorize test items using the category validity and cue validity of features that were unrelated to background knowledge, even though they also used background knowledge for properties that were related to the background knowledge. Thus, his subjects did not simply ignore the predictiveness of the knowledgeunrelated features when there was background knowledge available. However, Wisniewski did not directly assess the effects of background knowledge on how well subjects learned the structural information about the categories.

In short, many experiments have shown that background knowledge plays an important role in how categories are learned. The availability of background knowledge dramatically changes subjects' performance on various tasks, such as categorization and typicality judgments. However, it is not clear how background knowledge affects what subjects learn specifically about the structure of the categories.

The present experiments addressed this question of what is learned about a category that is strongly related to background knowledge. To investigate this, we focused on the degree to which subjects are sensitive to feature frequency. Feature frequency is often considered to be an important factor in determining the structure of peoples' categories (see, e.g., Barsalou, 1985; Kellogg, 1981). Indeed, some notion of feature frequency underlies many category learning models. Generally, the more often a feature occurs in a target category and the less often it occurs in other categories, the more highly weighted that feature is in the category representation (Hampton, 1979; Rosch \& Mervis, 1975; Smith \& Medin, 1981). Thus, feature frequency is an important variable in its own right. Many past studies of categorization have manipulated frequency of features or items in order to investigate their effects on learning or typicality (e.g., Barsalou, Huttenlocher, \& Lamberts, 1998; Nosofsky, 1988; Rosch \& Mervis, 1975). We, however, will be interested in frequency judgments about the features themselves, as a measure of what subjects have learned (see also Spalding \& Ross, 1994, Experiment 3).
As mentioned above, Murphy and Allopenna (1994) varied the frequency of features in their categories and found that this variable had little effect on typicality and categorization judgments about the features when the category was related to integrative knowledge. Thus, this was fairly strong support for the empirical insensitivity hypothesis. However, it is not clear from their results whether the subjects had not learned about feature frequency or simply did not use the information in making their judgments. For example, it is possible that subjects knew perfectly well that some features were much less frequent than others, but this point may have seemed irrelevant to making a typicality decision when there was integrative information available. For example, a feature that seems to be a good property of a jungle vehicle might have been rated as typical, even if it seldom occurred (cf. Barsalou's, 1985, notion of ideals as a determinant of typicality). Thus, it is not necessarily the case that subjects did not learn the frequency information, even if they did not appear to use it in typicality and categorization judgments.

The present experiments attempted to discover how the presence of background knowledge during category acquisition affects what subjects learn about feature frequency itself, by directly asking subjects to estimate feature frequency. In particular, the present experiments were meant to discover whether background knowledge decreases subjects' sensitivity to differences in feature frequency. The experiments all made use of a similar procedure. In general, easily integrated categories (integrated condition) were compared with categories that were difficult to integrate (nonintegrated condition) that had the same statistical structure. Within each category, there were some high-frequency features and some low-frequency features. In Experiments 1 and 2, the subjects were asked to estimate the frequency with which the features occurred in the set of exemplars. In Experiment 3, the subjects were asked to judge the typicality of the features. The subjects' sensitivity to the differences between highand low-frequency features was of primary interest in revealing how background knowledge affects what is learned.

\section{EXPERIMENT 1}

Subjects were taught a pair of categories and then asked to estimate the frequency with which each feature occurred in the set of instances. In one condition, the category's features could be integrated using general domain knowledge; in the other, the categories made sense, but the features were not easily interrelated. Subjects estimated the frequency of both relevant and irrelevant features. Relevant features were those that were predictive of one of the two categories, and irrelevant features were those that were not predictive (i.e., they occurred equally often in both categories). Relevant features were like those for which subjects showed empirical insensitivity in Murphy and Allopenna (1994) and so might be expected to show the same insensitivity in the present ex- 
periment. The irrelevant features were not predictive of category membership and were not related to the integrative knowledge. Thus, the irrelevant features provided a control, in that frequency estimates for the irrelevant features should not be affected by background knowledge. For each experiment, we conducted an overall analysis of the frequency estimates of both types of feature as well as more specific tests to assess the sensitivity to the difference between high- and low-frequency relevant features.

\section{Method}

Subjects. The subjects were 36 University of Illinois undergraduates who participated for course credit.

Materials. The subjects learned to distinguish two categories, each consisting of 10 exemplars. There were two feature conditions, integrated and nonintegrated. Features in the integrated condition were easily integrated into an overall knowledge structure for each category; features in the nonintegrated condition, although they did not contradict or conflict with each other, were not easily integrated into an overall knowledge structure. Table 1 shows the features of the vehicle categories (the materials were adapted from Murphy \& Allopenna, 1994). The integrated categories in Table 1 can be understood as jungle and arctic vehicles (the integrated categories in the animal domain could be understood as some types of predator and prey animals, and the integrated categories in the building domain could be understood as buildings that exist underwater or that float above the ground). We designed these categories so that they could be interpreted via domain knowledge yet would not match any particular real category. The posttest described after Experiment 3 tests this assumption. The nonintegrated categories had properties typical of vehicles but did not evoke any more particular knowledge, and the properties themselves were not strongly related. The integrated and nonintegrated conditions used the same formal category structure (see Appendix A for an abstract design of the categories).

Table 1

Example Materials for Experiment 1

\begin{tabular}{|c|c|c|}
\hline & Category 1 & Category 2 \\
\hline \multicolumn{3}{|c|}{ Integrated Condition } \\
\hline 20 & Green & White \\
\hline 12 & Made in Africa & Made in Norway \\
\hline 12 & Lightly insulated & Heavily insulated \\
\hline 12 & Drives in jungles & Drives on glaciers \\
\hline 12 & Has wheels & Has treads \\
\hline 4 & Convertible & Nonconvertible \\
\hline \multicolumn{3}{|c|}{ Irrelevant features } \\
\hline & \multirow{3}{*}{\multicolumn{2}{|c|}{$\begin{array}{l}\text { License plate in front, License plate in back } \\
\text { Uses gasoline, Uses diesel } \\
\text { Four door, Two door }\end{array}$}} \\
\hline & & \\
\hline & & \\
\hline
\end{tabular}

\begin{tabular}{rll} 
& \multicolumn{2}{c}{ Nonintegrated Condition } \\
20 & Green & White \\
12 & Nonradial tires & Radial tires \\
12 & Airbag & Automatic seatbelts \\
12 & Automatic transmission & Manual transmission \\
12 & Cloth seat covers & Vinyl seat covers \\
4 & Convertible & Nonconvertible \\
\multicolumn{2}{c}{ Irrelevant features } \\
20 & License plate in front, License plate in back \\
12 & Uses gasoline, Uses diesel \\
4 & Four door, Two door &
\end{tabular}

Note-Irrelevant features are listed in the middle column because they were not predictive of either category. The number preceding each dimension indicates the number of instances that included that dimension (each value has a frequency that is half of the frequency of the dimension).
The materials represented three different domains (vehicles, animals, and buildings). Values of six dimensions were relevant to the categories (occurring in only one of the categories), and values of three dimensions were irrelevant to the categories (occurring equally often in the two categories). Within the relevant set of dimensions, one dimension occurred on all 20 instances, one dimension occurred on only 4 instances, and the other four dimensions each occurred on 12 instances. Within the irrelevant set of dimensions, one dimension occurred on all 20 instances, one occurred on 12 instances, and one occurred on 4 instances. Note that each dimension had two values (which we often call features), so value (feature) frequencies were half the dimension frequencies: 10,6 , and 2 . So, in Table 1 , the " 20 " next to the row listing "Green" and "White" indicates that green and white each occurred 10 times. The only differences between the integrated and nonintegrated conditions were the four relevant dimensions that each occurred on 12 instances.

As can be seen in Table 1, the irrelevant dimensions were the same for the integrated and nonintegrated conditions (within domains; in this case, vehicles). In addition, the highest frequency relevant dimension was the same, as was the lowest frequency relevant dimension. In this way, comparisons of the effect of background knowledge on subjective frequency were not confounded with item

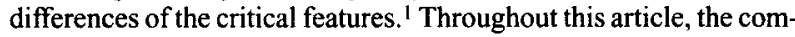
parisons across groups (the knowledge manipulation) were always of the exact same features.

Each instance included the highest frequency relevant dimension and the highest frequency irrelevant dimension. The 10 instances in each category contained all possible combinations of three of the four middle-frequency relevant features ( 4 instances) and all possible combinations of two of the four middle-frequency relevant features ( 6 instances); Appendix A shows the exact configurations of each item. Thus, all instances included at least three relevant features and, at most, two irrelevant features. Each instance was printed on an index card. The features were printed on the cards in random orders.

Procedure. A standard category learning paradigm was used. Each subject learned a single pair of categories that were either integrated or nonintegrated, in only one of the domains (vehicles, animals, or buildings). (So, the subjects might learn to categorize the arctic and jungle vehicles but would not learn any other categories.) The subjects were told that they would be seeing some descriptions of imaginary objects that belonged to two categories, one called Dax and one called $\mathrm{Kez}$, and that they should try to learn as much as they could about the objects and the Dax and Kez categories. They were told that there would be some later tests but were not told the nature of those tests. The subjects were given the cards in a random order and asked to guess the category membership of each one. The experimenter told the subjects whether they were correct and then let them study the card for a total of $10 \mathrm{sec}$. Each subject went through the set of 20 instances for three blocks. Pilot studies indicated that most subjects in the integrated condition discovered the knowledge structure (i.e., the subjects understood how the features could be integrated) at the end of the first study block or during the second, and most subjects in the nonintegrated condition were able to learn the categories fairly well with three study blocks. Given that the subjects estimated frequencies, it was important that all subjects receive the same number of exposures to the instances.

After three blocks, the subjects were given a sheet of paper that listed all of the features (in a random order) that occurred in the cards read by the subjects. The subjects estimated, for each feature, the number of instances that had included that feature. The subjects were told that there were 20 items in all, so no feature could have occurred on more than 20 cards.

\section{Results and Discussion}

On average, the subjects in both the integrated condition and the nonintegrated condition responded correctly on $99 \%$ of the items during the third learning block. 
Clearly, the subjects learned the categorical distinction quite well.

As mentioned earlier, only the estimates for the highand low-frequency features were analyzed, because only they can be compared across the integrated and nonintegrated conditions without confounding the comparison with feature differences. The results are presented in Table 2 . These frequency estimates were analyzed using a 2 $\times 2 \times 2$ analysis of variance (ANOVA), with knowledge condition (integrated vs. nonintegrated) as a between-subjects factor and feature relevance (relevant vs. irrelevant) and frequency (high vs. low) as within-subjects factors. As expected, high-frequency features had higher subjective frequencies than low-frequency features $(9.3$ vs. 4.7 ; actual frequencies 10 and 2) $\left[F(1,34)=156.18, M S_{\mathrm{e}}=4.84\right.$, $p<.05]$. Clearly, the subjects were sensitive to the actual frequency of the features. Additionally, features that were statistically relevant to the categorization were (incorrectly) judged to be more frequent than features that were statistically irrelevant to the categorization (7.5 vs. 6.4) $\left[F(1,34)=4.71, M S_{\mathrm{e}}=8.52, p<.05\right]$. It should be noted, however, that we cannot be absolutely sure that the relevant-irrelevant difference was due only to statistical relevance. As can be seen in Table 1, comparison between relevant and irrelevant features does involve different features (as does the high-frequency-low-frequency difference), so it is possible that there may be some aspect of the features themselves (salience, perhaps) that accounts for this difference, rather than statistical relevance per se. Given the primary goal of manipulating background knowledge, this featural difference was unavoidable. The relevant-irrelevant difference was approximately the same across the three different domains used in these experiments, however, suggesting that it is unlikely to be purely based on salience of particular features. The effect of frequency was also rather consistent across domains. No other main effects or interactions approached significance.

Of particular interest, of course, is the degree to which the subjects in the two conditions were sensitive to the difference between the high- and low-frequency relevant features (note that exactly the same features are being compared across the integrated and nonintegrated conditions). According to the empirical insensitivity hypothesis, and extrapolating from Murphy and Allopenna's (1994) results with typicality ratings, the integrated group should show lower sensitivity to frequency differences. The results, however, were in the opposite direction: The subjects were slightly more sensitive to the difference

Table 2

Mean Subjective Frequencies From Experiment 1

\begin{tabular}{lccccc}
\hline & \multicolumn{2}{c}{ Integrated } & & \multicolumn{2}{c}{ Nonintegrated } \\
\cline { 2 - 3 } \cline { 5 - 6 } & Relevant & Irrelevant & & Relevant & Irrelevant \\
\hline High frequency & 10.4 & 8.8 & & 9.7 & 8.1 \\
Low frequency & 4.5 & 5.2 & & 5.3 & 3.7 \\
Difference & 5.9 & 3.6 & & 4.4 & 4.4
\end{tabular}

Note-Actual frequency of high-frequency features was 10 ; actual frequency of low-frequency features was 2 . between high- and low-frequency relevant features in the integrated condition (mean difference $=5.9$ ) than in the nonintegrated condition (mean difference $=4.4$ ), although this difference was not reliable $[t(34)=1.43, p=.15]$. (The actual frequency difference was 8.0.) We did not expect any difference between groups in frequency sensitivity for the irrelevant features, and none occurred $[t(34)<1]$.

Thus, Experiment 1 provided no evidence for the empirical insensitivity hypothesis. Both groups of subjects showed a strong effect of feature frequency in their estimates, and, if anything, the results suggest that knowledge was improving people's sensitivity to feature frequency.

\section{EXPERIMENT 2}

Given that Experiment 1 did not find the empirical insensitivity reported by Murphy and Allopenna (1994), we felt that it was important to attempt to replicate this result, at the same time eliminating one possible problem. In Experiment 1, the high-frequency relevant feature could be used to perfectly partition all the instances into the two categories. We included this highest frequency feature in order to be sure that there was a large difference between high- and low-frequency features. However, because natural categories do not generally have such defining features, it was important to be sure that the lack of empirical insensitivity observed in Experiment 1 was not due to the defining feature. In addition, the fact that the highly frequent features occurred on every instance of a category could have caused the subjects to "anchor" their frequency estimates for the high-frequency features at the top of the allowable frequency estimate range that they were given, thus decreasing the likelihood of finding significant differences between the conditions. That is, if subjects in both conditions anchored their estimates for the highest frequency features at 10 , then differences in sensitivity could only result from differences at the low-frequency end, thereby decreasing the potential differences in sensitivity across the knowledge conditions. Clearly, the subjects were quite sensitive to frequency overall, so such a large range of feature frequencies was not necessary. Additionally, there was no defining feature in the Murphy and Allopenna experiments that demonstrated empirical insensitivity, making comparisons between the present results and theirs somewhat problematic. Thus, in Experiment 2 , the high-frequency features occurred on only 6 of the 10 items in the category.

\section{Method}

Sixty undergraduates from the University of Iowa participated for course credit. The materials were adapted from Experiment 1. An abstract design is provided in Appendix B. There were five relevant dimensions with frequency 12 and one relevant dimension with frequency 4 . There was one irrelevant dimension with frequency 20 , one with frequency 12 , and one with frequency 4 . The 10 instances of each category were created by taking all possible combinations of three of the five relevant dimensions with frequency 12 . The highest frequency irrelevant dimension, of course, occurred on every instance. The two lowest frequency dimensions 
were added in such a way that every instance had five features. In this design, there was no single dimension that could be used to correctly partition all the instances.

As in Experiment 1, there were two critical dimensions that were used in both the integrated condition and the nonintegrated condition to allow us to compare frequency effects across conditions unconfounded by item differences. For the purposes of this experiment, we refer to the critical relevant dimension as the high-frequency dimension, even though its frequency was equal to that of four other relevant dimensions. (By the same token, we refer to the frequency 12 irrelevant dimension that was matched across conditions as high frequency, although there was an irrelevant dimension that was more frequent.) High frequency and low frequency are intended to describe the relative frequency of only the critical dimensions, which were used in both conditions. The procedure was identical to Experiment 1.

\section{Results and Discussion}

On average, the subjects in both the integrated condition and the nonintegrated condition responded correctly on more than $97 \%$ and $93 \%$, respectively, of the items during the third study block. The lack of a defining feature probably made the categorical distinction in Experiment 2 more difficult than in Experiment 1, accounting for the small drop in accuracy. Nevertheless, it is clear that the subjects learned the categories reasonably well with three study blocks.

The results are presented in Table 3. As in Experiment 1 , high-frequency features were judged to be more frequent than low-frequency features ( 7.5 vs. 4.6 ; actual frequencies 6 and 2) $\left[F(1,58)=90.1, M S_{\mathrm{e}}=5.5, p<\right.$ $.05]$. In addition, relevant features were (incorrectly) given marginally higher frequency estimates than irrelevant features (6.3 vs. 5.8$)\left[F(1,58)=3.0, M S_{\mathrm{e}}=4.2, p<.10\right]$. There was also a marginal three-way interaction between knowledge, feature relevance, and frequency $[F(1,58)=$ $\left.3.0, M S_{\mathrm{e}}=3.2, p<.10\right]$, which is related to the primary question of interest-whether the relevant features would show empirical insensitivity.

As before, we analyzed the estimates for the critical relevant dimensions separately. The actual differences between high- and low-frequency relevant features were 4 in the present experiment. The subjects in the integrated condition gave estimates extremely close to this value (mean difference $=3.9$ ) and, in fact, were reliably more sensitive to this difference than were the subjects in the nonintegrated condition (mean difference $=1.9)[t(58)=$ $2.4, p<.05]$. The availability of background knowledge clearly made these subjects more sensitive to the frequency difference for the relevant features, rather than less sen-

Table 3

Mean Subjective Frequencies From Experiment 2

\begin{tabular}{lccccc}
\hline & \multicolumn{2}{c}{ Integrated } & & \multicolumn{2}{c}{ Nonintegrated } \\
\cline { 2 - 3 } \cline { 5 - 6 } \cline { 5 - 6 } & Relevant & Irrelevant & & Relevant & Irrelevant \\
\hline High frequency & 8.3 & 7.1 & & 7.2 & 7.5 \\
Low frequency & 4.4 & 4.2 & & 5.3 & 4.6 \\
Difference & 3.9 & 2.9 & & 1.9 & 2.9
\end{tabular}

Note-Actual frequency of high-frequency features was 6 ; actual frequency of low-frequency features was 2 . sitive - contrary to the empirical insensitivity hypothesis but consistent with the trend found in Experiment 1. As expected, the control condition (irrelevant features) showed no difference at all between the integrated and nonintegrated conditions (both means $=2.9$ ).

\section{EXPERIMENT 3}

At this point, it seems clear that the empirical insensitivity hypothesis simply does not hold for feature frequency. If anything, background knowledge seems to help people learn about the feature frequencies. Although Murphy and Allopenna (1994) found that background knowledge led to less sensitivity to frequency in categorization and typicality judgments, we find that background knowledge leads to more sensitivity to frequency when frequency is directly estimated by the subjects. It is possible that there is some fundamental difference between how subjects make use of what they have learned about the categories in the categorization/typicality task on the one hand and the feature frequency estimation task on the other. However, there are some small procedural and stimulus differences between the present experiments and those of Murphy and Allopenna. Thus, it is not certain that the difference between typicality judgments and feature frequency estimation accounts for the difference between our results and theirs. Experiment 3 used the present stimuli and procedure in an attempt to replicate Murphy and Allopenna's finding that subjects with background knowledge are less sensitive to frequency differences when making typicality judgments.

Experiment 3 used the same items and learning task as Experiment 2. However, at test, subjects made categorization decisions for each feature and then rated its typicality in the chosen category, rather than making frequency estimates. If the original Murphy and Allopenna (1994) findings of empirical insensitivity are not replicated, this will suggest that some aspect of the procedure or stimulus structure used in the present experiments is responsible for our failure to find such an effect. If, on the other hand, subjects are more sensitive to frequency differences in the nonintegrated condition (i.e., empirical insensitivity), this will provide strong evidence that typicality judgments differ from frequency judgments in a fundamental way, such that there is empirical insensitivity for typicality ratings, but not for frequency judgments, when background knowledge is available.

\section{Method}

Subjects. Twenty-four University of Illinois undergraduates participated for course credit or for pay.

Materials. The instances were the same as those in Experiment 2 (described in Appendix B). For the typicality rating task, a sheet of paper was used with a list of all the features presented in the experiment along with a blank for the subject to indicate category membership and another blank for the subject to rate the feature's typicality (for the category chosen) on a scale of 1 to 7 .

Procedure. The study procedure was identical to that of Experiment 2. After learning the categories, the subjects were given the typicality test sheet and instructed for each feature to indicate to 
which category that feature belonged. They were also instructed to rate each feature for its typicality in the category to which they had just assigned it on a scale of 1 (not at all typical) to 7 (extremely typical). The exact instructions follow:

\begin{abstract}
Now that you have learned something about these two kinds of things, we would like to ask you some questions about them. We will give you a sheet of paper with a list of properties on it. For each property, we would like you to do two things. First, write down the name of the category that the property goes with. That is, write "Dax" or "Kez," depending on which one it belongs with. Second, we would like you to rate the typicality of the property to that category. How typical, usual, or representative is it of Dax or Kez (whichever your answer was)? Rate the typicality on a 1 to 7 scale, where 1 means that the feature is not at all typical, and 7 means that the property is extremely typical of that category. Use intermediate numbers for intermediate levels of typicality. Try to use the whole scale - not just one or two numbers.
\end{abstract}

\section{Results and Discussion}

The subjects in both conditions learned the categories quite well, responding with $95 \%$ accuracy in the third study block. In this experiment, only the ratings given for the relevant features were analyzed, because it was unclear how to count the typicality for features that do not have a correct category assignment. If a feature was assigned to the incorrect category, the feature's typicality was multiplied by -1 , creating a typicality scale ranging from -7 to +7 . This was done because a typicality judgment for a feature, even in the wrong category, still provides important information. That is, if the learner thinks that a feature that was actually predictive of one category is highly typical in the other category, this reveals worse accuracy than if he or she thought the feature was only a little typical of the other category (see, e.g., Nosofsky, 1988, and Wisniewski, 1995, for similar treatments of typicality data). For each subject, the two low-frequency features' typicality scores were averaged and then subtracted from the average typicality of the high-frequency features, exactly as the frequency differences were analyzed in the previous experiments. The difference scores for all subjects were entered into a one-way betweengroups ANOVA.

The results were much like the categorization and typicality results of Murphy and Allopenna (1994) and were unlike the patterns of Experiments 1 and 2. There was a nearly significant difference between the integrated and nonintegrated conditions $\left[F(1,22)=3.94, M S_{\mathrm{e}}=20.02\right.$, $p=.058]$, indicating that the subjects were more sensitive to frequency differences in the nonintegrated condition than in the integrated condition. As expected, the subjects in the nonintegrated condition showed a reliable effect of feature frequency. The mean typicality difference between high- and low-frequency features was 3.67 and was reliably greater than zero $[t(11)=3.14, p<$ $.01]$. However, the difference for the subjects in the integrated condition was essentially nonexistent. The mean typicality difference was $.04[t(11)=.03$, n.s. $]$. Although the subjects made few categorization errors, those that were made followed the same pattern of greater sensitivity to frequency differences in the nonintegrated condition. The subjects in the nonintegrated condition made only two incorrect category assignments on high-frequency features and seven on low-frequency features, but the subjects in the integrated condition made five on highfrequency features and three on low-frequency features.

At this point, we should clear up one potential concern. The typicality judgment task differs from the frequency judgment task in that the typicality judgment task requires a judgment with respect to one particular category, but the frequency judgment task requires only an overall judgment of the frequency of the feature across both categories. Therefore, the subjects in the earlier experiments might have known the overall frequencies without knowing which category a feature was associated with (although they had to know the categories for at least some of the features in order to perform so well in the learning phase of the experiments). Thus, the typicality judgment task might show insensitivity to frequency because the subjects only knew overall frequencies, but not which features were associated with which categories. The present data, however, clearly indicate that the subjects did know which category the features belonged to. Overall, the subjects in the integrated condition correctly classified the target features on about $92 \%$ of the test trials. Given that the learning procedure was identical to the earlier experiments, it seems clear that the subjects did indeed know which category the target features were associated with, as well as knowing (even if sometimes not using) the frequency information.

In summary, the subjects in the nonintegrated condition were quite sensitive to feature frequency in making typicality judgments, but those in the integrated condition were relatively insensitive. Obviously, this is the empirical insensitivity found by Murphy and Allopenna (1994) and is very different from the pattern found in Experiments 1 and 2 when the subjects were asked directly about feature frequency. Figure 1 illustrates the contrast between the two effects.

Thus, we have something of a puzzle. When subjects do not have available background knowledge, their judgments of typicality are highly sensitive to what they have learned about feature frequency. However, when they have available background knowledge, they learn the frequency differences better, but they do not seem to use what they know about feature frequency in assigning typicality to the features.

\section{STIMULUS POSTTESTS}

One question that might arise in considering the results of the present experiments is whether the subjects are making their judgments with reference to actual realworld objects and whether this might account for the obtained results. The first stimulus posttest assessed whether the subjects thought that the integrated categories were descriptions of actual real-world objects. The second stimulus posttest investigated whether real-world frequency differences among the features could account for the results. As we noted earlier, the exact same features were used in the two conditions (integrated and nonintegrated), so pure feature frequency could not account for our results. 


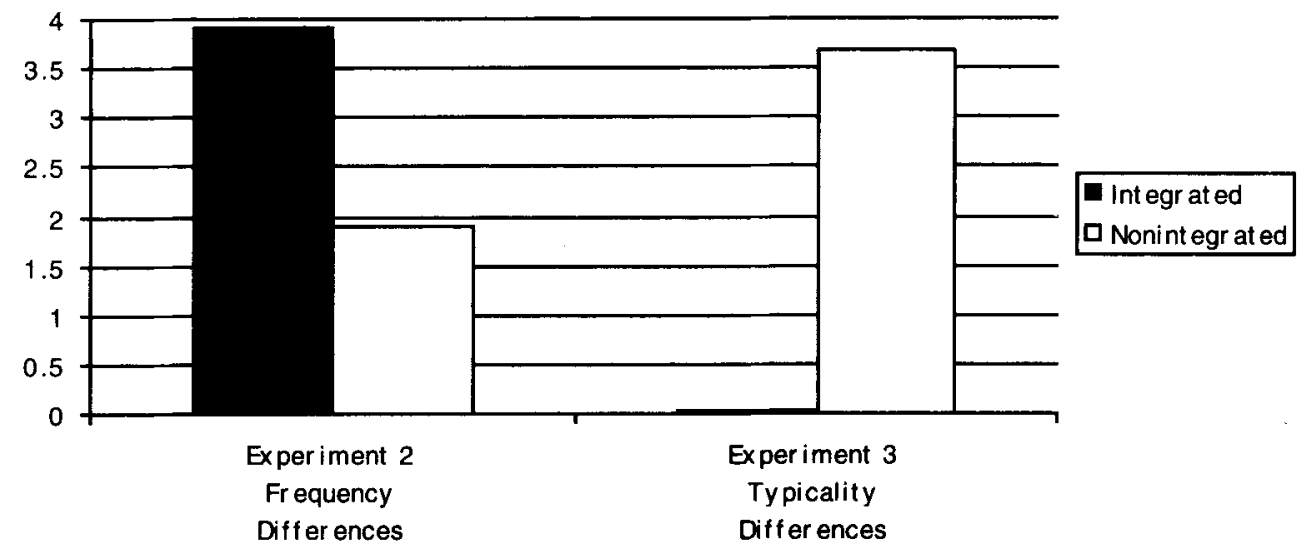

Figure 1. Differences between frequency estimates for high- and low-frequency relevant features as a function of knowledge condition in Experiment 2 (the actual difference equals 4) and differences between typicality judgments for high- and low-frequency relevant features as a function of knowledge condition in Experiment 3. Larger differences indicate more sensitivity to the difference between high- and lowfrequency features.

However, the features might have differed in their perceived frequency within categories of that type. For example, perhaps "green" (see Table 1) is thought to be a very frequent feature in the context of jungle vehicles but not in the context of vehicles as a whole, and this artifactually caused the differences in frequency judgments across knowledge conditions. The second test allowed us to test this interpretation of our results.

\section{Method: Stimulus Posttest 1}

This test was directly based on a test conducted by Murphy and Allopenna (1994). The subjects were given the relevant features of our integrated categories, our nonintegrated categories, and four sets of features describing real-world categories (chair, bird, pants, and saw, drawn from the feature listings of Rosch, Mervis, Gray, Johnson, \& Boyes-Braem, 1976). Subjects should be able to identify the real-world categories fairly easily. The question is whether the experimental categories are also identified as being real categories and, most importantly, whether the integrated categories are more likely to be seen as real than the nonintegrated ones.

The relevant features for the categories in the earlier experiments were used. Each set consisted of six features, listed in an arbitrary order. Each subject judged three integrated categories, three nonintegrated categories, and four actual object categories. A given subject judged only one of each pair of categories (e.g., a subject that judged the "arctic vehicle" would not judge the "jungle vehicle," and vice versa). The subjects were told that they would be reading sets of features, some of which would describe familiar objects, such as an apple or a lawyer, and some would not. They were asked to decide whether each set of features described a familiar object and, if it did, to write down the name of the object. Sets of features were presented in random orders. Sixty-seven University of Iowa undergraduates participated for course credit.

\section{Results and Discussion: Stimulus Posttest 1}

The main results concern the percentage of subjects who identified categories of each type as familiar. The means for the three conditions were $29 \%$ for the integrated categories, $57 \%$ for the nonintegrated categories, and $98 \%$ for the actual object categories. Almost all of the subjects identified the actual objects as being familiar (and cor- rectly identified the intended category), but less than a third identified the integrated categories as being familiar. Importantly, the integrated categories were identified as real even less often than the nonintegrated categories $[t(66)=6.08, p<.01]$. Furthermore, even when the integrated categories were judged to be familiar, there was little agreement about what the familiar category was. For example, the animal that is placid and slow, eats plants, has flat teeth and a furry tail, and lives in groups was identified as a cow, a deer, a hippopotamus, a caterpillar, a beaver, and a duck-billed platypus, among others. Clearly, the integrated categories did not reliably correspond to actual real-world categories.

\section{Method: Stimulus Posttest 2}

This test was designed to investigate whether subjects thought there were differences in real-world frequency of the critical features, depending on whether they encountered the critical features in the context of the integrated or nonintegrated categories. (We are grateful to the reviewers for pointing out this possibility to us.) In this test, the subjects were shown the set of features used in an experimental category and were asked to rate the frequency of the critical features for all real-world objects similar to the experimental category.

The same sets of features for the experimental categories were used as in the first stimulus posttest. As in the first posttest, the subjects rated only one of each category pair. Each subject saw three integrated categories and three nonintegrated categories. No subject ever rated a particular critical feature for more than one category. Each set of six features was presented on a separate page, along with the instructions for the rating task. Below this, each page had a rating question for each of the two critical features. Each critical feature rating question had the same form: "On a scale of 1 to 7 , how frequent do you think the feature _____ is among all the REAL WORLD objects similar to the imaginary object described above?" The subjects were told that the set of features described a kind of imaginary object and that we wanted them to judge the frequency of the designated features among all the real-world objects similar to the description. The subjects rated the features on a scale of 1 to $7(7=$ very frequent ). If they felt there were no real-world objects that were similar to the description, they gave the feature a 0 . Fifty University of Iowa undergraduates participated for course credit. None had participated in the earlier experiments or in the first stimulus posttest. 


\section{Results and Discussion: Stimulus Posttest 2}

The integrated high-frequency features summed rating was 10.42 , integrated low frequency was 11.68 , nonintegrated high frequency was 10.26 , and nonintegrated low frequency was 11.06 . There was no effect of category type $\left[F(1,49)<1, M S_{\mathrm{e}}=18.9\right]$. Our low-frequency features were rated slightly higher than the high-frequency features $\left[F(1,49)=4.78, M S_{\mathrm{e}}=11.1, p<.05\right]$. Most importantly, there was no interaction $\left[F(1,49)<1, M S_{\mathrm{e}}=10.8\right]$. Thus, there is no hint of a difference in the real-world frequency of the features depending on whether the features were presented in the integrated or nonintegrated categories. The one difference found-low-frequency features rated higher than high-frequency features-apparently did not have much effect in the main experiments, since highfrequency features obtained much higher frequency estimates in Experiments 1 and 2. The subjects seem to have been relying primarily on observed frequency for their frequency judgments, as instructed, rather than relying on knowledge of similar real categories.

\section{GENERAL DISCUSSION}

The present experiments provide a more complete, but also more complex view of the phenomenon of empirical insensitivity, as proposed by Murphy and Allopenna (1994). First, Experiment 3 replicated their result, in that when the features were related by integrative knowledge, frequent features were not thought to be more typical than infrequent features. Yet, when integrative knowledge was not available, frequency was a significant predictor of typicality. However, Experiments 1 and 2 showed a pattern in the opposite direction for frequency judgments themselves: For the integrated categories, subjects showed a greater difference (and one closer to the correct value) between frequent and infrequent features than for the nonintegrated categories. (This difference approached significance in Experiment 1 and was significant in Experiment 2. However, our main interest here is that the direction is opposite to that found for typicality judgments in Experiment 3 and in Murphy \& Allopenna, 1994.)

These results provide an interesting perspective on the question of what is learned when people use background knowledge in acquiring a category. First, contrary to the proposal of Murphy and Allopenna (1994), it is clear that subjects learned both the integrative knowledge and the detailed statistical structure of the category, as revealed in the frequency estimates. Although subjects could have performed perfectly in the category learning task by using only the integrative knowledge (e.g., "Category $\mathrm{A}$ is the arctic vehicle"), their later performance shows that they learned a considerable amount about the individual features. In fact, it seems that the knowledge actually helped subjects to learn about the features in the category (i.e., the reliably greater sensitivity to frequency in Experiment 2).

So, in terms of the two proposals mentioned in the introduction, the evidence seems to favor the second one, that knowledge is not replacing or short-circuiting the learning about specific details but is instead actually mak- ing that learning more efficient. If this is the case, then it seems likely that knowledge is having important effects at encoding of the properties, as suggested by many views of background knowledge (e.g., Murphy \& Medin, 1985; see also Wisniewski, 1995, for a comparison of encoding and retrieval effects of knowledge).

That conclusion, however, should not lead us to assume that all the effects of knowledge are happening at encoding, since the typicality results are clearly due to later retrieval/judgment stages. We know from the frequency measures that subjects are learning the statistical properties of the features when they have knowledge. We also know that feature frequency is an important predictor of the rated typicality and categorization of the feature for subjects without knowledge (in Experiment 3 and in Murphy \& Allopenna, 1994). Nonetheless, when the category was related to integrative knowledge, subjects did not use this frequency information to judge typicality, as shown in Experiment 3 (the difference score was virtually 0 ). The subjects simply did not seem to think that the frequency differences were relevant to typicality judgments when they had knowledge, even though they were perfectly aware of those differences. Thus, at test, subjects seem to make their typicality decisions based on the fit of the feature to the integrative knowledge. Of course, if they do not have integrative knowledge, then subjects fall back on their knowledge of the feature frequencies.

This result can be related to the more general claim that people often believe that there is an underlying essence or reason for categories to be the way that they are. Medin and Ortony (1989) proposed that subjects believe that there are underlying causes that lead categories to have the properties they do, even though subjects do not know what these causes are for every category. Subjects treat these causes as being the core or true nature of the category (see also Bloom, 1996). There is empirical evidence for this position as well. Keil's (1989) "transformation" studies, in which underlying biological principles overrode surface appearance in older children's and adults' categorization decisions about natural kinds, provide evidence for this view. Specifically with respect to typicality judgments, Barsalou (1985) showed that category ideals greatly determined typicality ratings, both for natural categories and for artificial materials in which he directly manipulated the ideals (see also Ahn, Kim, Lassaline, \& Dennis, 1999). Similarly, Wisniewski (1995) showed that when an item had features functionally related to the integrative knowledge underlying a category, the item was categorized as being a member of that category even when its properties were statistically more related to another category. In all these cases, people used the underlying function, motivation, or cause of the category to judge typicality, independently of observed empirical structure.

Our results can be described in a similar vein. Once subjects integrated the features, they may have thought that this constituted the real nature of the category, which was more important than the presence or absence of any of the superficial properties. A feature such as "convertible" is consistent with the idea of a jungle vehicle but 
not the idea of an arctic vehicle, and so subjects rate it as very typical of the jungle vehicle category, even though it was seldom observed (see Table 1). Thus, once subjects integrate the features into a consistent knowledge structure (e.g., arctic vehicles) for the category, then the consistency of features to the knowledge structure determines their category judgments much more than the statistical properties do, even though the subjects have also learned the statistical properties. In a sense, then, the relation of a feature to the integrative knowledge trumps the statistical aspects of the feature's relation to the category (as in Wisniewski, 1995, as well).

However, we must ask what it is about the typicality judgment task that allows the knowledge to trump the statistical information, or, alternatively, what it is about the frequency judgment task that stops background knowledge from trumping the statistical information. The most obvious difference is that the typicality task requires evaluation of the feature with respect to the category (e.g., how typical is this feature of the Dax category?), whereas the frequency judgment does not. Spalding and Ford (1998) investigated the role of background knowledge in sensitivity to feature frequency in speeded categorization (requires evaluating with respect to the category) and in speeded recognition (does not require evaluation with respect to the category). They found speeded categorization results that were very similar to the present results for typicality judgments and speeded recognition results that were similar to the present results for frequency judgments. In Spalding and Ford, the subjects were assigned to one of the two test conditions after an identical learning phase. Thus, the subjects in the two different testing conditions presumably learned the same things about the categories, and it was the test tasks that determined how background knowledge affected their performance. That is, subjects who showed little sensitivity to frequency in categorization would have shown sensitivity in recognition, and vice versa, since subjects were randomly assigned to test tasks after a common learning task. This is also probably a reasonable assumption for the present experiments, since the materials and learning procedures for Experiments 2 and 3 were identical.

Thus, one explanation that is consistent with both the present data and the data of Spalding and Ford (1998) is that background knowledge leads to the creation of an integrated knowledge structure that is used for tasks that require evaluation with respect to the category. However, when the task does not require evaluation with respect to the category, then the integrated knowledge structure may not be accessed. Clearly, though, the integrated knowledge structure does not replace the more detailed knowledge of the statistical structure. Instead, it may be that the integrated knowledge structure is formed in addition to the statistical structure and that either may be accessed, perhaps to different degrees, by a particular task. Presently, it seems that evaluation with respect to the category is important in determining whether or not the background knowledge "takes over." There may well be many other factors. Further research will be needed to determine exactly how the decision task determines what knowledge is accessed.
One particularly novel aspect of the present results is that background knowledge might actually aid the acquisition of more detailed category information. Past experiments on background knowledge in category learning have focused almost entirely on typicality or categorization judgments (Barsalou, 1985; Murphy \& Allopenna, 1994; Wattenmaker et al., 1986; Wisniewski, 1995), which generally conflate encoding and judgment effects. In the present experiments, we were able to show that subjects do acquire the statistical information, even if they do not feel that it is important to use in typicality judgments. Other work in our laboratories is also finding evidence that knowledge may be benefiting the learning of more detailed category structure (Kaplan \& Murphy, 1999; Spalding \& Phillips, 1998b). Spalding and Ford (1998) have recently found analogous results in memory tests. Subjects with background knowledge were not very sensitive to feature frequency when categorizing features but were sensitive to frequency when recognizing features. However, subjects without background knowledge were more sensitive to frequency when categorizing features than when recognizing features. As we mentioned in the introduction, there is a tradition in the memory literature that suggests that use of background knowledge will lead to inaccuracies in encoding of specific details. For example, the main dependent measure of the use of knowledge for some studies is whether subjects will make false alarms to script- or schema-related events that did not actually occur (e.g., Bower et al., 1979; Bransford \& Franks, 1971). However, more recent research, with careful separation of verbatim and gist memory, has suggested that background knowledge or script information does not necessarily lead to the loss of more specific information (Craik et al., 1994; Murphy \& Shapiro, 1994).

Perhaps the main theoretical question remaining, then, is how to explain the apparently better learning of the featural information when knowledge is present. Most models of feature frequency and categorization do not include any component of background knowledge, and so they cannot make specific predictions about the present results. However, one can attempt to extend these models to see if one of their mechanisms might account for some of the results. One typical means of explaining differences between features is to focus on the strength of encoding of the feature or the weight of its dimension (e.g., Kruschke, 1992; Medin \& Schaffer, 1978; Nosofsky, 1986, 1988; Nosofsky \& Kruschke, 1992; see also Hintzman, 1988, particularly the simulation of Rowe's, 1974 , data). Kruschke (1993) claims that some of the effects of background knowledge can be dealt with in the same way. In our case, this could be translated into higher dimensional weighting for knowledge-related features, causing them to be rated as being more frequent.

The problem with these explanations is that they make the same predictions for the knowledge effect and for statistical relevance. But our data do not support the similarity of these mechanisms nor the suggestion that background knowledge should lead to a main effect on frequency judgments. In Experiments 1 and 2, we found that 
statistical relevance causes an overall increase in frequency judgments, but not a greater sensitivity to frequency differences among stimuli. Knowledge, on the other hand, led to greater sensitivity to frequency differences but no overall increase in frequency estimates. Spalding and Ford (1998) also found that there was no main effect advantage for background knowledge in memory for features. It is clear that if the same mechanism is used to explain statistical predictability and background knowledge, this proposal cannot explain the different pattern of results we have found. Seemingly, background knowledge allows people to be more sensitive to feature frequency, but without increasing the strength of encoding in such a way that the features overall would be judged to be higher frequency (or be recalled or recognized more easily, as shown in Spalding \& Ford, 1998).

In short, there are two aspects to the way knowledge influences the featural processing. By relating each feature to the integrative knowledge, it provides a conceptual connection to the knowledge structure, and this is what is apparently critical in the typicality judgments (where frequency has little effect). However, knowledge also affects the encoding of the category members in such a way that the representation of the features can be used to estimate frequency more accurately. It is still something of a puzzle as to why knowledge effects result in greater sensitivity to frequency differences without increasing overall frequency estimates. Future research will have to address this surprising finding.

\section{Conclusion}

Our results have shown that knowledge does not make category learners less sensitive to the detailed category structure. Rather than replacing featural learning, knowledge seems to enhance it, as revealed by more accurate frequency estimates. However, the knowledge does overrule feature frequency information when the task is to relate features to a category representation, as in typicality and categorization judgments. Thus, knowledge is used as an aid to learning rather than replacing the need for more specific category learning.

\section{REFERENCES}

AhN, W.-K., Kim, N. S., Lassaline, M. E., \& Dennis, M. J. (1999). Causal status effect as a determinant of feature centrality. Manuscript submitted for publication.

BARSALOU, L. W. (1985). Ideals, central tendency, and frequency of instantiation as determinants of graded structure in categories. Journal of Experimental Psychology: Learning, Memory, \& Cognition, 11, 629-654.

Barsalou, L. W., Huttenlocher, J., \& Lamberts, K. (1998). Basing categorization on individuals and events. Cognitive Psychology, 36, 203-272.

BLoom, P. (1996). Intention, history, and artifact concepts. Cognition, 60, 1-29.

BOWER, G. H., BLACK, J. B., \& TURNER, T. J. (1979). Scripts in memory for text. Cognitive Psychology, 11, 177-220.

BRANSFORD, J. D., \& Franks, J. J. (1971). Abstraction of linguistic ideas. Cognitive Psychology, 2, 331-350.

BREWER, W. F., \& TREYENS, J. C. (1981). Role of schemata in memory for places. Cognitive Psychology, 13, 207-230.
Cralk, F. I. M., Moscovitch, M., \& McDowd, J. (1994). Contributions of surface and conceptual information to performance on implicit and explicit memory tasks. Journal of Experimental Psychology: Learning, Memory, \& Cognition, 20, 864-875.

HAMPTON, J. A. (1979). Polymorphous concepts in semantic memory. Journal of Verbal Learning \& Verbal Behavior, 18, 441-461.

HEIT, E. (1997). Knowledge and concept learning. In K. Lamberts \& D. Shanks (Eds.), Knowledge, concepts and categories (pp. 7-41). Hove, U.K.: Psychology Press.

HiNTZMAN, D. L. (1988). Judgments of frequency and recognition memory in a multiple-trace memory model. Psychological Review, 95, 528-551.

KAPLAN, A. S., \& MURPHY, G. L. (1999). The acquisition of category structure in unsupervised learning. Memory \& Cognition, 27, 699-712.

KEIL, F. (1989). Concepts, kinds, and cognitive development. Cambridge, MA: MIT Press.

KeLLOGG, R. T. (1981). Feature frequency in concept learning: What is counted? Memory \& Cognition, 9, 157-163.

Komatsu, L. K. (1992). Recent views of conceptual structure. Psychological Bulletin, 112, 500-526.

KRUSCHKE, J. (1992). ALCOVE: An exemplar-based connectionist model of category learning. Psychological Review, 99, 22-44.

KRUSCHKE, J. (1993). Three principles for models of category learning. In G. Nakamura, R. Taraban, \& D. L. Medin (Eds.), The psychology of learning and motivation (Vol. 29, pp. 57-90). New York: Academic Press.

Medin, D. L. (1989). Concepts and conceptual structure. American Psychologist, 44, 1469-1481.

Medin, D. L., \& Ortony, A. (1989). Psychological essentialism. In S. Vosniadou \& A. Ortony (Eds.), Similarity and analogical reasoning (pp. 179-195). New York: Cambridge University Press.

Medin, D. L., \& Schaffer, M. M. (1978). Context theory of classification learning. Psychological Review, 85, 207-238.

MurPHy, G. L. (1993). Theories and concept formation. In I. Van Mechelen, J. Hampton, R. Michalski, \& P. Theuns (Eds.), Categories and concepts: Theoretical views and inductive data analysis (pp. 173200). London: Academic Press.

MurPhy, G. L., \& Allopenna, P. D. (1994). The locus of knowledge effects in concept learning. Journal of Experimental Psychology: Learning, Memory, \& Cognition, 20, 904-919.

MURPhy, G. L., \& KaPLAN, A. S. (1997). Category learning with minimal knowledge. In M. Ramscar, U. Hahn, E. Cambouropolos, \& H. Pain (Eds.), Proceedings of SimCat 1997: An interdisciplinary workshop in similarity and categorization (pp. 165-171). Edinburgh University, Department of Artificial Intelligence.

MurPhy, G. L., \& MEDin, D. L. (1985). The role of theories in conceptual coherence. Psychological Review, 92, 289-316.

MurPHY, G. L., \& ShAPIRO, A. M. (1994). Forgetting of verbatim information in discourse. Memory \& Cognition, 22, 85-94.

MurPhy, G. L., \& SPalding, T. L. (1995). Knowledge, similarity, and concept formation. Psychologica Belgica, 35, 127-144.

NosOFSKY, R. M. (1986). Attention, similarity, and the identificationclassification relationship. Journal of Experimental Psychology: General, 115, 39-57.

Nosofsky, R. M. (1988). Similarity, frequency, and category representations. Journal of Experimental Psychology: Learning, Memory, \& Cognition, 14, 54-65.

NosofSKY, R. M., \& KRUSChKE, J. K. (1992). Investigations of in exemplar-based connectionist model of category learning. In D. L. Medin (Ed.), The psychology of learning and motivation (Vol. 28, pp. 207-250). New York: Academic Press.

PAZZANI, M. J. (1991). Influence of prior knowledge on cone spt acquisition: Experimental and computational results. Journal if Experimental Psychology: Learning, Memory, \& Cognition, 17, 416-432.

Rosch, E., \& Mervis, C. B. (1975). Family resemblance: Studies in the internal structure of categories. Cognitive Psychology, 7, 573-605.

Rosch, E., Mervis, C. B., Gray, W. D., Johnson, D. M., \& BoyesBraEm, P. (1976). Basic objects in natural categories. Cognitive Psychology, 8, 382-439.

RowE, E. J. (1974). Depth of processing in a frequency judgment task. Journal of Verbal Learning \& Verbal Behavior, 13, 638-643. 
Smith, E. E., \& MEdin, D. L. (1981). Categories and concepts. Cambridge, MA: Harvard University Press.

SPALDING, T. L., \& FoRD, M. A. (1998). Background knowledge and memory for category features. Manuscript submitted for publication.

SpaldinG, T. L., \& MurPhy, G. L. (1996). Effects of background knowledge on category construction. Journal of Experimental Psychology: Learning, Memory, \& Cognition, 22, 525-538.

Spalding, T. L., \& Phillips, V. L. (1998a). Background knowledge use in category formation: Roles of the learning and decision tasks. Manuscript submitted for publication.

Spalding, T. L., \& Phillips, V. L. (1998b). What happens when features mismatch background knowledge about a category? Evidence from typicality, categorization, and recognition judgments. Manuscript submitted for publication.

SpaldinG, T. L., \& Ross, B. H. (1994). Comparison-based learning: Effects of comparing instances during category learning. Journal of Experimental Psychology: Learning, Memory, \& Cognition, 20, 1251-1263.

Wattenmaker, W. D., Dewey, G. I., Murphy, T. D., \& Medin, D. L.
(1986). Linear separability and concept learning: Context, relational properties, and concept naturalness. Cognitive Psychology, 18, 158-194. WISNIEWSKI, E. (1995). Prior knowledge and functionally relevant features in concept learning. Journal of Experimental Psychology: Learning, Memory, \& Cognition, 21, 449-468.

\section{NOTE}

1. As can be seen from Table 1, it was possible to choose two dimensions from the integrated categories that did not by themselves evoke enough background knowledge for the subjects to integrate all the features of the instances into a unified knowledge structure. That is, vehicles that are green are related to the jungle vehicle knowledge structure, and convertibles are more likely to be used in a hot weather climate than in an arctic climate. But these two features alone, when mixed with a number of unrelated features, would not be sufficient to suggest the jungle vehicle knowledge structure to the subjects. This assumption is verified by the results of Experiments 2 and 3, which found reliably different judgments of these features in the integrated and nonintegrated categories.

APPENDIX A

Abstract Design for Experiment 1

\begin{tabular}{|c|c|c|c|c|c|c|c|c|c|c|c|c|c|c|c|c|c|}
\hline \multicolumn{9}{|c|}{ Category 1} & \multicolumn{9}{|c|}{ Category 2} \\
\hline D1 & $\mathrm{D} 2$ & D3 & D4 & D5 & D6 & D7 & D8 & D9 & D1 & D2 & D3 & D4 & D5 & D6 & D7 & D8 & D9 \\
\hline 1 & 0 & 1 & 1 & 1 & 0 & 1 & 0 & 0 & 2 & 0 & 2 & 2 & 2 & 0 & 2 & 0 & 0 \\
\hline 1 & 1 & 1 & 1 & 0 & 0 & 2 & 0 & 0 & 2 & 2 & 2 & 2 & 0 & 0 & 1 & 0 & 0 \\
\hline 1 & 1 & $i$ & 0 & 1 & 0 & 1 & 0 & 1 & 2 & 2 & 2 & 0 & 2 & 0 & 2 & 0 & 2 \\
\hline 1 & 1 & 0 & 1 & 1 & 0 & 2 & 0 & 2 & 2 & 2 & 0 & 2 & 2 & 0 & 1 & 0 & 1 \\
\hline 1 & 0 & 1 & $i$ & 0 & 1 & 1 & 1 & 0 & 2 & 0 & 2 & 2 & 0 & 2 & 2 & 2 & 0 \\
\hline 1 & 1 & 1 & 0 & 0 & 1 & 2 & 1 & 0 & 2 & 2 & 2 & 0 & 0 & 2 & 1 & 2 & 0 \\
\hline 1 & 0 & 1 & 0 & 1 & 0 & 1 & 1 & 0 & 2 & 0 & 2 & 0 & 2 & 0 & 2 & 2 & 0 \\
\hline 1 & 1 & 0 & 1 & 0 & 0 & 2 & 2 & 0 & 2 & 2 & 0 & 2 & 0 & 0 & 1 & 1 & 0 \\
\hline 1 & 0 & 0 & 1 & 1 & 0 & 1 & 2 & 0 & 2 & 0 & 0 & 2 & 2 & 0 & 2 & 1 & 0 \\
\hline 1 & 1 & 0 & 0 & 1 & 0 & 2 & 2 & 0 & 2 & 2 & 0 & 0 & 2 & 0 & 1 & 1 & 0 \\
\hline
\end{tabular}

Note--Each dimension has two values ( 1 or 2$)$. A 0 indicates no value for that dimension. D1 is the high-frequency relevant dimension. D2-D5 are the middle-frequency relevant dimensions. D6 is the low-frequency relevant dimension. D7 is the highfrequency irrelevant dimension. D8 is the middle-frequency irrelevant dimension. D9 is the low-frequency irrelevant dimension.

APPENDIX B

Abstract Design for Experiments 2 and 3

\begin{tabular}{|c|c|c|c|c|c|c|c|c|c|c|c|c|c|c|c|c|c|}
\hline \multicolumn{9}{|c|}{ Category 1} & \multicolumn{9}{|c|}{ Category 2} \\
\hline D1 & D2 & D3 & D4 & D5 & D6 & D7 & D8 & D9 & D1 & D2 & D3 & D4 & D5 & D6 & D7 & D8 & D9 \\
\hline 1 & 1 & 1 & 0 & 0 & 0 & 1 & 0 & 1 & 2 & 2 & 2 & 0 & 0 & 0 & 2 & 0 & 1 \\
\hline$i$ & 1 & 0 & 1 & 0 & 0 & 2 & 0 & 2 & 2 & 2 & 0 & 2 & 0 & 0 & 1 & 0 & 2 \\
\hline 1 & 1 & 0 & 0 & 1 & 1 & 1 & 0 & 0 & 2 & 2 & 0 & 0 & 2 & 2 & 2 & 0 & 0 \\
\hline 1 & 0 & 1 & 1 & 0 & 1 & 2 & 0 & 0 & 2 & 0 & 2 & 2 & 0 & 2 & 1 & 0 & 0 \\
\hline 1 & 0 & 1 & 0 & 1 & 0 & 1 & 1 & 0 & 2 & 0 & 2 & 0 & 2 & 0 & 2 & 2 & 0 \\
\hline 1 & 0 & 0 & 1 & 1 & 0 & 2 & 1 & 0 & 2 & 0 & 0 & 2 & 2 & 0 & 1 & 2 & 0 \\
\hline 0 & 1 & 1 & 1 & 0 & 0 & 1 & 1 & 0 & 0 & 2 & 2 & 2 & 0 & 0 & 2 & 2 & 0 \\
\hline 0 & 1 & 1 & 0 & 1 & 0 & 2 & 2 & 0 & 0 & 2 & 2 & 0 & 2 & 0 & 1 & 1 & 0 \\
\hline 0 & 1 & 0 & 1 & 1 & 0 & 1 & 2 & 0 & 0 & 2 & 0 & 2 & 2 & 0 & 2 & 1 & 0 \\
\hline 0 & 0 & 1 & 1 & 1 & 0 & 2 & 2 & 0 & 0 & 0 & 2 & 2 & 2 & 0 & 1 & 1 & 0 \\
\hline
\end{tabular}

Note--Each dimension has two values ( 1 or 2$)$. A 0 indicates no value for that dimension. D1 is the high-frequency relevant dimension. D6 is the low-frequency relevant dimension. D2-D5 are the frequency 12 relevant dimensions. D7 is the frequency 20 irrelevant dimension. D8 is the high-frequency irrelevant dimension. D9 is the low-frequency irrelevant dimension. 